\title{
Intervenciones psicológicas en la ONCE durante la crisis de la covid-19
}

\section{Psychological care provided by the ONCE during the covid-19 crisis}

\section{González García, R. Pallero González}

\section{Resumen}

Este artículo revisa las intervenciones realizadas por los profesionales de la psicología de la ONCE durante la crisis de la covid-19. Se expone en qué consistieron esas intervenciones, y se analizan su incidencia y sus resultados. Las intervenciones psicológicas fueron tanto personales y directas como psicoeducativas, tanto telepsicológicas como presenciales, tanto individuales como grupales, tanto con adultos como con niños y adolescentes y sus familias. Desde marzo a julio de 2020, la mayoría de las intervenciones se realizaron en la modalidad de telepsicología, combinando la atención por videoconferencia con la atención por teléfono. En lo que a las intervenciones por videoconferencia se refiere, seis de cada diez se realizaron con personas de entre 30 y 60 años, en gran medida porque los menores de 30 años no fueron objetivo preferente de intervención, y porque los mayores de 60 años presentan una baja competencia digital. De modo complementario, durante ese período se pusieron a disposición de los profesionales de la psicología y de los afiliados diversos materiales de tipo psicoeducativo, como documentos, vídeos, archivos multimedia..., en los que se ofrecían orientaciones para hacer frente a la situación. La telepsicología, que justo antes de la irrupción de la covid-19 se empezaba a implantar en la ONCE, se ha convertido en una vía más de intervención, habiendo experimentado un crecimiento exponencial a lo largo del año 2020. Debemos estar atentos a que este incremento del uso de la telepsicología, especialmente de la realizada por videoconferencia, no deje al margen a las personas con menos destrezas en el manejo de las tecnologías de la información y comunicación. Tanto en el caso de las intervenciones psicológicas como en el de las psicoeducativas, sería importante poder conocer su incidencia y el valor que le atribuyen sus usuarios. 


\title{
Palabras clave
}

Intervenciones psicológicas. Telepsicología. Psicoeducación. Competencia digital. Trabajo en red. Covid-19.

\begin{abstract}
This article reviews the care provided by the ONCE's psychologists during the covid-19 crisis. It describes what such care involved and analyses incidence and results. Adults, children, teenagers and their families received psychological support both personally / directly and in the form of psychoeducation, remotely and in person, via both individual and group therapy. From March to July 2020, most care was provided remotely, combining teleconferencing and telephone contact. Six of every ten videoconferences were held with people between the ages of 30 and 60 , primarily because the under-30s were not a priority target and the over-60s tended to lack the necessary digital competence. Supplementarily, during that period ONCE professionals and affiliates had access to psychoeducational material including documents, videos and multimedia files containing guidelines on how to confront the situation. Telepsychology, which had begun to be introduced in the ONCE shortly before the onset of covid-19, became a standard procedure, exhibiting exponential growth throughout 2020. Policies must nonetheless be implemented to ensure that people with a limited command of information and communication technologies are not left by the wayside in the wake of this more frequent deployment of telepsychology, particularly as regards teleconferencing. Ascertainment of the incidence and value attributed by users to both psychological care and psychoeducational measures is of particular importance.
\end{abstract}

\section{Key words}

Psychological action. Telepsychology. Psychoeducation. Digital competence. Networking. Covid-19.

\section{Introducción}

El servicio de apoyo psicosocial de la ONCE tiene como eje principal de sus intervenciones el procurar el ajuste a la discapacidad visual de las personas afiliadas a la institución, y ello tanto si son personas adultas como si están dentro del ámbito educativo. Las intervenciones orientadas al ajuste a la discapacidad visual pue- 
den ser individuales, familiares y grupales. Hasta 2020 dichas intervenciones eran habitualmente presenciales, aunque ocasionalmente se realizaban intervenciones telefónicas. Sin embargo, la geografía de muchas zonas de España, caracterizada por la dispersión de la población y las escasas infraestructuras de transporte, dificulta en muchos casos esa atención presencial, generando diferencias entre unos territorios y otros, entre unos afiliados y otros, lo que llevó a la ONCE a plantearse la conveniencia de incorporar nuevos modos de prestar este servicio. Por ello, y dada la creciente presencia de las tecnologías de la información y comunicación (TIC) en nuestras vidas, la ONCE tomó la decisión de iniciar la prestación de servicios de apoyo psicosocial por medio de videoconferencia, de modo auxiliar y complementario a la atención presencial directa.

Para ello, en octubre de 2019 la totalidad de los profesionales de la psicología de la ONCE recibieron una formación intensiva sobre intervención telepsicológica, sus despachos fueron dotados de la tecnología necesaria para su implantación y se constituyó un grupo de trabajo sobre telepsicología. Simultáneamente, el Departamento de Autonomía Personal, con la participación del mencionado grupo de trabajo, desarrolló unas instrucciones de uso de las tecnologías de la comunicación aplicadas a las terapias psicológicas en la ONCE.

Aunque por aquel entonces se desconocía, ahora sabemos que en esos momentos ya había empezado a circular entre los humanos un nuevo virus, al que la Organización Mundial de la Salud (OMS) aún tardaría unos meses en ponerle nombre: coronavirus de tipo 2 causante del síndrome respiratorio agudo severo (SARS-CoV-2). Un nombre demasiado largo, que pronto se empezó a acotar, para ser conocido simplemente como coronavirus, el cual causaba una enfermedad nueva, a la que la OMS llamó covid-19 (OMS, s.f.).

Pocos meses después, nada más empezar febrero de 2020, cuando el coronavirus todavía no era noticia de portada en los medios de comunicación, cuando no se llegaba a 25000 casos en el mundo, siendo menos de 200 de ellos fuera de China, y solo 2 en España (OMS, 5 de febrero de 2020), se abrió la posibilidad del uso de la videoconferencia para la prestación de los mencionados servicios de apoyo psicosocial en la ONCE.

En ese momento, la puesta en marcha de la telepsicología en la ONCE se planteaba como algo progresivo, para intervenciones preferentemente individuales, respondien- 
do a unas instrucciones muy precisas, siguiendo un proceso de toma de decisiones muy estructurado, alternando sesiones presenciales y telemáticas (aunque optando de modo preferente por las primeras), utilizando una plataforma tecnológica de videoconferencia específica (Zoom), accediendo a dicha plataforma utilizando las licencias de las que dispone el Departamento de Autonomía Personal de la ONCE. La elección de la plataforma Zoom, que tanto protagonismo ha alcanzado durante el último año, fue realizada por Dirección Técnica de Sistemas y Tecnologías de la Información de la ONCE, buscando garantizar la eficacia tecnológica y el cumplimiento del Reglamento general de protección de datos (RGPD).

Pero en solo un mes la situación cambió radicalmente. En marzo de 2020 la OMS calificó la situación provocada por la covid-19 como pandemia internacional (OMS, 11 de marzo de 2020), y en España un Real Decreto (RD 463/2020, de 14 de marzo) declaró el estado de alarma. En los días siguientes, la ONCE suspendió prácticamente la totalidad de su actividad laboral presencial, y puso en marcha equipos básicos de atención a las personas más vulnerables, formados por psicólogos, trabajadores sociales, instructores en tecnología y braille y responsables de voluntariado, además de a los profesores de los equipos de apoyo educativo.

Estas circunstancias provocaron que el servicio de apoyo psicosocial que oferta la ONCE - que, como se ha dicho, tiene como eje principal de sus intervenciones procurar el ajuste a la discapacidad visual de las personas afiliadas a la mismatuviese que suspender muchas de ellas, al tiempo que hubo que hacer cambios en los objetivos y la metodología del resto. Pero, además, provocó la aparición de nuevas demandas de atención psicológica no relacionadas con el ajuste a la discapacidad visual, sino con el ajuste a la situación de pandemia, con sintomatología como ansiedad, depresión, alteraciones del sueño, rumia, miedo a la enfermedad, duelo por fallecimiento de familiares... Ante esta situación, la ONCE dio instrucciones a sus profesionales de la psicología para dar respuesta directa a estas demandas, sin tener que derivarlas a servicios ajenos a la ONCE como, en otras circunstancias, hubiera sido lo propio. Todas estas intervenciones se regularon por medio de un documento, alojado en el área de trabajo compartido de los profesionales de la psicología de la ONCE, ubicada en la intranet de la ONCE para uso de sus empleados (PortalONCE), titulado Intervención psicológica en el contexto ocasionado por la covid-19, el cual se complementó con la modificación de las instrucciones que regulaban el uso de la telepsicología, para adaptarlas a la situación de crisis.

González, L., y Pallero, R. (2021). Intervenciones psicológicas en la ONCE durante la crisis de la covid-19. RED Visual: Revista Especializada en Discapacidad Visual, 77, 68-91. https://doi.org/10.53094/WILG7697. 
Las intervenciones realizadas por los profesionales de la psicología de la ONCE durante la crisis de la covid-19, desarrolladas con la supervisión del Departamento de Autonomía, podemos agruparlas en tres grandes categorías:

- Intervenciones psicológicas individuales, mediante las que se prestó apoyo psicológico personal y directo a los afiliados a la ONCE que presentaban sintomatología psicológica reactiva bien ante su pérdida de funcionalidad visual, bien ante la situación de pandemia, bien ante ambas circunstancias. Entre marzo y junio de 2020 estas intervenciones se realizaron por teléfono y/o por videoconferencia, medios que se mantuvieron posteriormente, aunque, progresivamente, se fueron recuperando las intervenciones presenciales.

- Intervenciones psicológicas grupales, mediante las que se prestó apoyo psicológico, en formato de grupos reducidos, a los afiliados a la ONCE que presentaban malestar genérico y preocupación ante la situación de pandemia. Dichas intervenciones se empezaron a realizar a partir de julio de 2020, tanto por videoconferencia como presencialmente.

- Intervenciones psicoeducativas (Anderson et al., 1980; Authier, 1977; Lemes y Ondere, 2017), mediante las que se prestó apoyo psicológico indirecto a los afiliados a la ONCE, poniendo a su disposición una serie de guías de apoyo emocional, con las que orientarlos para hacer frente a las consecuencias emocionales de la situación de pandemia.

Estas intervenciones fueron apoyadas con diversa documentación que se puso a disposición de todo el colectivo profesional, seleccionada entre las publicaciones que los colegios de psicología de toda España, así como otras instituciones públicas y privadas, fueron realizando sobre la situación de pandemia y confinamiento.

Como se ha señalado, durante el confinamiento domiciliario y la posterior desescalada (es decir, entre el 14 de marzo y el 14 de junio de 2020), estas intervenciones tuvieron que realizarse, necesariamente, utilizando medios propios de la telepsicología (videoconferencias, llamadas telefónicas, publicaciones en Internet...). Ello provocó una inercia que, unida a las recomendaciones sanitarias, ha hecho que la telepsicología se haya convertido en una vía significativa de intervención desde entonces, tanto dentro como fuera de la ONCE, suponiendo un número muy importante de las intervenciones de los profesionales de la psicología. 
Pero volvamos la vista atrás: veamos detalladamente en qué han consistido ( $y$ en buena medida siguen consistiendo) las intervenciones psicológicas en la ONCE durante la crisis de la covid-19.

\section{Fuentes de información}

Las fuentes de las que hemos obtenido la información que recopilamos en este artículo han sido variopintas, al tratarse de una revisión tanto cuantitativa como cualitativa de la atención prestada por los profesionales de la psicología de la ONCE durante la crisis de la covid-19.

Las fuentes utilizadas para obtener datos cuantitativos fueron:

- El análisis de las fichas de solicitud de conexiones de Zoom realizadas por los profesionales de la psicología de la ONCE al Departamento de Autonomía Personal, entre el 1 de febrero y el 31 de diciembre de 2020.

- Las respuestas de los profesionales de la psicología de la ONCE a un cuestionario sobre el uso de la telepsicología durante el año 2020, elaborado por el grupo de trabajo sobre telepsicología, y cuyas respuestas fueron recogidas entre el 18 y el 29 de enero de 2021.

Las fuentes utilizadas para obtener información cualitativa fueron:

- La revisión de los registros de actividad del grupo de trabajo sobre telepsicología.

- La revisión de los registros de actividad del grupo de trabajo sobre intervención con menores y jóvenes en la desescalada y la vuelta a la escolarización.

- La revisión de la documentación que, sobre apoyo a la intervención psicológica durante la crisis de la covid-19, fue subida al área de trabajo compartido de los profesionales de la psicología de la ONCE en PortalONCE durante el año 2020.

- La revisión de la documentación de carácter psicoeducativo que, para el abordaje de la crisis de la covid-19, fue subida a la intranet de la ONCE para uso de sus afiliados (ClubONCE) durante el año 2020. 


\section{Intervenciones de los profesionales de la psicología de la ONCE}

Todos los datos que se ofrecen en este apartado, salvo que expresamente se indique lo contrario, se refieren a situaciones acaecidas entre 1 de febrero y el 31 de diciembre de 2020.

\subsection{Intervenciones directas}

La plantilla de profesionales de la psicología de la ONCE en el momento de inicio de la pandemia estaba compuesta por 40 profesionales de la psicología, de los cuales 34 estuvieron activos durante el confinamiento domiciliario por el coronavirus y la posterior desescalada (recordemos: entre el 14 de marzo y el 14 de junio de 2020), lo que implica que, durante ese período, el $85 \%$ de estos profesionales de la ONCE realizaron intervenciones telepsicológicas, puesto que esa era la única alternativa de intervención.

A partir del 15 de junio de 2020, una vez que todos los profesionales de la psicología de la ONCE se reincorporaron a la actividad laboral en sus puestos de trabajo, y hasta el final del año 2020, prácticamente la totalidad de ellos (en concreto, el 96,7\%) realizaron intervenciones telepsicológicas.

Durante 2020, y en lo que a la telepsicología por videoconferencia se refiere, 18 de los 39 profesionales de la psicología de la ONCE han hecho uso de alguna de las licencias de Zoom de las que dispone el Departamento de Autonomía Personal de la ONCE, lo que supone un $46,2 \%$ de los mismos. A través de esas licencias se agendaron 574 sesiones de telepsicología por videoconferencia. Resulta muy interesante desagregar este dato, y lo haremos en tres períodos, los cuales vienen determinados por los acontecimientos relacionados con la crisis de la covid-19:

- Entre el 5 de febrero (fecha de la puesta en marcha de la telepsicología en la ONCE) y el 15 de marzo (día posterior a la declaración del estado de alarma), se agendaron 3 sesiones, lo que supone una media de 2 sesiones mensuales.

- Entre el 16 de marzo y el 14 de junio (períodos de confinamiento y de desescalada), se agendaron 126 sesiones, lo que supone una media de 42 sesiones mensuales. 
- Entre el 15 de junio (fecha de inicio de la «nueva normalidad») y el final del año 2020, se agendaron 448 sesiones, lo que supone una media de 69 sesiones mensuales.

El número de usuarios distintos atendidos utilizando esas licencias ha sido de más de 120 , lo que supone una media de casi cinco sesiones por usuario.

Además de las licencias de Zoom del Departamento de Autonomía Personal de la ONCE, muchos profesionales de la psicología de la ONCE utilizaron tanto las licencias de sus centros de trabajo como sus propias cuentas personales de Zoom, además de otras plataformas con acceso a videoconferencia, como WhatsApp, Google Meet, Microsoft Teams o Google Duo. Lamentablemente, todas estas intervenciones no han podido ser cuantificadas.

Pero, como hemos indicado, no todas las sesiones de telepsicología se realizaron por videoconferencia. La mayoría de las intervenciones (en concreto, el 58,6\%) se realizaron combinando la atención por videoconferencia con la atención por teléfono, el $27,6 \%$ se realizaron fundamentalmente por teléfono, mientras que solo el $13,8 \%$ de las intervenciones se realizaron fundamentalmente por videoconferencia.

En cuanto al número de usuarios atendido, el $69 \%$ de los profesionales de la psicología de la ONCE realizó intervenciones telepsicológicas con más de 20 usuarios distintos cada uno de ellos. A falta de datos más precisos, ese dato nos permite hacer una estimación de que, durante 2020, fueron atendidos, utilizando la telepsicología, nunca menos de 500 usuarios distintos.

Conforme establecen las instrucciones de uso de la telepsicología en la ONCE, la gran mayoría de esas intervenciones han sido única o mayoritariamente individuales ( $34,5 \%$ y $41,4 \%$, respectivamente), aunque una cuarta parte de ellas (en concreto un $24,1 \%)$ han sido tanto individuales como grupales.

En cuanto al tipo de problemática atendida durante la crisis del coronavirus, los profesionales de la psicología refieren que, en el $82,8 \%$ de los casos, ha sido, prácticamente a partes iguales: tanto ajuste a la discapacidad visual como problemática derivada de la situación de pandemia.

En cuanto al grado de satisfacción de los profesionales de la psicología con el uso que, hasta la fecha, se está haciendo de la telepsicología, su valoración media es de 
4,14 puntos sobre 5. Aún no se ha tenido la oportunidad de valorar la satisfacción de los usuarios, pero la estimación de los profesionales de la psicología es que es muy similar a la suya propia.

Veamos ahora cuáles están siendo las características de los usuarios de la telepsicología durante esta crisis de la covid-19. En lo que a su género se refiere, el 58,6\% de los profesionales de la psicología de la ONCE señala que fueron por igual hombres y mujeres, pero es de destacar que el 37,9\% señala que fueron, mayoritariamente, mujeres. En lo que a su edad se refiere, el 62,1\% fueron mayoritariamente de media edad (entre 30 y 60 años), el 24,1\% mayoritariamente personas mayores (de más de 60 años) y el 13,8\% mayoritariamente jóvenes (menores de 30 años).

En cuanto al uso de la videoconferencia, uno de los principales problemas que refleja la encuesta es la falta de competencia digital de los usuarios de la telepsicología, algo que cabía esperar, si tenemos en cuenta que el 45,6\% de los afiliados a la ONCE tienen 65 o más años, un segmento de edad que, según datos de una encuesta del Instituto Nacional de Estadística (INE), hace un menor uso de las TIC (INE, 2020). Así, nos encontramos con que el $37,9 \%$ de los profesionales de la psicología de la ONCE percibe la competencia digital de sus usuarios como baja, mientras que solo el $3,4 \%$ la valora como alta, si bien la mayoría (el 58,6\%) estima que la competencia de sus usuarios afiliados es media.

\subsection{Intervenciones indirectas}

Con el fin de objetivar lo más posible estos resultados, hemos decidido vincularlos a los «productos» surgidos de la actividad de los profesionales de la psicología de la ONCE durante la crisis de la covid-19.

Entre marzo y julio de 2020 varios grupos de trabajo, compuestos por psicólogos y psicólogas de la ONCE, y encabezados y coordinados por los departamentos de Autonomía Personal y de Atención Educativa, recibieron el encargo de compilar y elaborar una serie de documentos de apoyo psicológico para el afrontamiento de la crisis de la covid-19. Los destinatarios de dichos materiales serían tanto los afiliados a la ONCE y sus familias, en calidad de usuarios finales de los mismos, como los propios profesionales de la psicología de la ONCE, en su rol de mediadores para su uso con los afiliados. Pasemos a ver cuáles fueron esos materiales. 


\subsubsection{Documentos subidos al área de trabajo compartido de los profesionales de la psicología de la ONCE en PortalONCE}

Un grupo de profesionales de la psicología de la ONCE ${ }^{1}$ creado al efecto fue el encargado de elaborar, por un lado, una guía de recomendaciones psicológicas para afiliados y, por otro lado, un protocolo de intervención psicológica, ambos enfocados en el momento que se estaba viendo, debido a la covid-19. La finalidad de estos materiales, que podríamos encuadrar dentro de un modelo de intervención psicoeducativo, era poner a disposición de los profesionales de la psicología de la ONCE algunos materiales de referencia para orientar sus intervenciones, ya fueran estas presenciales, por medio de la telepsicología o combinadas. Su uso, en todo caso, quedaba a discreción de dichos profesionales.

El primero de esos documentos, la Guía de recomendaciones psicológicas para personas afiliadas en el período de desconfinamiento es, como su descriptivo nombre indica, una guía con recomendaciones psicológicas para afrontar la salida del confinamiento. Dicha guía está estructurada en cuatro partes:

- En la primera se proponen unas reflexiones sobre lo que ha pasado y lo que sigue pasando, es decir, tratando de hacer una revisión retrospectiva para, seguidamente, focalizarse en el presente de lo que significa en nuestras vidas la covid-19 y sus consecuencias.

- En la segunda se presenta una relación de estados emocionales, presentados por parejas contrapuestas (tristeza-alegría, miedo-confianza...), que, de un modo u otro, han aparecido en nosotros durante la crisis del coronavirus. Unas emociones que es importante reconocer en uno mismo y en las personas más cercanas a nosotros, y que hay que aprender a llevar de una manera más sana. Para que el manejo de esas emociones sea más sencillo, cada pareja se presenta asociada al título de una película, a un refrán, a una frase hecha..., y a una conocida canción.

- En la tercera se plantea un ejercicio para reflexionar sobre el estado emocional de cada uno tras el confinamiento: cómo nos ha afectado, cómo hemos resuelto

1 Compuesto (por orden alfabético) por Mercedes Castillo Gómez, Félix Dorado Gómez, Pura García Andújar, Luis González García, Charo Ledesma Gallego, Maricarmen Martínez, Maru Martínez Zamora, Rafael Pallero González, Encarna Peláez Rivera y Vicky Puig Samaniego, con la supervisión de Carmen Millán Vera. 
o intentado resolver las situaciones que se nos fueron presentando, qué hemos descubierto, y qué hemos aprendido y cómo nos proyectamos hacia el futuro.

- Y en la cuarta se ofrecen una serie de recursos para relajarse y reducir tensiones.

Esta guía cuenta con una versión adaptada a lengua de signos española ${ }^{2}$ y con una versión breve. Todas ellas, además de poderse encontrar en el área de trabajo compartido de los profesionales de la psicología de la ONCE en PortalONCE, fueron también subidas a ClubONCE.

El segundo documento, el Protocolo de intervención psicológica para afrontar el desconfinamiento en adultos, propone un programa dual, de intervención preventiva y de intervención terapéutica (para casos severos de ansiedad, depresión, apatía, tristeza, desesperanza, duelo). La intervención preventiva, de carácter grupal, se estructura en ocho sesiones, mientras que la intervención terapéutica está diseñada para ser realizada en doce sesiones, nueve de ellas grupales, centradas en la intervención en situaciones de ansiedad y depresión, y tres sesiones complementarias, preferentemente individuales, centradas en situaciones de duelo, dirigidas a aquellos afiliados que hayan sufrido el fallecimiento de algún ser querido durante la pandemia por coronavirus. Tanto las intervenciones preventivas como las terapéuticas se cierran con dos sesiones más, pensadas para hacer una revisión de lo vivido y lo aprendido, y para proponerse nuevos planteamientos de futuro.

Este completo protocolo incluye, además, un modelo de entrevista inicial, un cuestionario de cribado, un cuestionario breve a administrar pre y post intervención, una prueba auditiva (para aplicar a usuarios con sordoceguera) y dos anexos con un banco de recursos para las sesiones preventivas y terapéuticas.

Además de estos dos documentos, se subieron a PortalONCE muchos otros materiales (documentos, vídeos, archivos multimedia - podcast-...), de uso público, elaborados por instituciones y personas ajenas a la ONCE, focalizados en esta situación de crisis, relacionados con la intervención psicológica en general y con la intervención en duelo en particular, y con recursos de regulación emocional.

2 Elaborada por la Unidad Técnica de Sordoceguera, Vicky Puig Samaniego y Raquel Alba Martín. 


\subsubsection{Documentos subidos a ClubONCE}

En el momento inicial, en pleno confinamiento domiciliario (es decir, entre mediados de marzo y mediados de junio de 2020), el grupo de trabajo sobre telepsicología en la $\mathrm{ONCE}^{3}$ preparó un documento titulado Recomendaciones psicológicas para afiliados, con recomendaciones para hacer frente al malestar emocional derivado de la situación de confinamiento: comprender la situación, evitar la sobreinformación, crear rutinas diarias, mantenerse en contacto, mantenerse activo, cuidar la forma física y la higiene, potenciar la ayuda mutua, favorecer un afrontamiento positivo y saber pedir ayuda.

Más adelante, una vez iniciada la desescalada, se subió a ClubONCE el documento citado en el epígrafe anterior, la Guía de recomendaciones psicológicas para personas afiliadas en el período de desconfinamiento, y sus versiones adaptada a lengua de signos española y breve.

También se subieron muchos otros materiales, de uso público, elaborados tanto por diversos colectivos profesionales de la ONCE como por instituciones y personas ajenas a la misma, relacionados con la autonomía personal y el uso de la tecnología, con el ocio y la cultura, con el bienestar físico y emocional, con la formación y el apoyo al empleo, orientados tanto a adultos como a menores y sus familias. Entre los materiales relacionados con el bienestar emocional, nos gustaría destacar los siguientes:

- El documento titulado Mantén tu tono emocional en la fase de confinamiento por el estado de alarma por el coronavirus, en el que se proponen dos estrategias generales de afrontamiento psicológico del coronavirus, se plantean distintos tipos de afrontamiento psicológico - según varias circunstancias vitales- y se ofrecen diez consejos para el afrontamiento psicológico del coronavirus.

- Tres grabaciones con ejercicios de relajación.

\subsubsection{Materiales para la intervención con niños y adolescentes, $y$ con sus familias}

Hasta ahora nos hemos referido únicamente a la intervención con personas adultas, pero ¿qué papel tuvo la intervención psicológica con los más pequeños de la casa?

3 Compuesto (por orden alfabético) por Almudena Cacho González, Félix Dorado Gómez, José Antonio García Regaña, Luis González García, Rafael Pallero González y Eva Vázquez Seoane, con la supervisión de Carmen Millán Vera. 
Es un hecho ampliamente reconocido que, durante la crisis generada por la covid-19, los niños y adolescentes y sus necesidades pasaron a un segundo plano: los colegios e institutos permanecieron con sus aulas vacías durante seis meses y la atención educativa pasó a ser por vía telemática, mientras que la atención a aspectos emocionales del desarrollo de los alumnos -y de su adaptación a las circunstancias- pasó, en casi todos los casos, a ser un asunto del que se ocuparon exclusivamente sus padres. Se ha destacar que la atención educativa del alumnado con discapacidad visual se mantuvo, tanto telemáticamente como con materiales adaptados, por parte de los profesores de apoyo de la ONCE y de las administraciones autonómicas.

El final de la desescalada vino a coincidir con el final del curso escolar. Aunque acababan de empezar las vacaciones escolares y faltaban más de dos meses para el inicio del curso 2020-2021, había que empezar a pensar en cómo abordar el inicio del mismo en septiembre. Para ello, la Dirección de Educación, Empleo y Braille de la ONCE encargó a un grupo de profesionales de la psicología ${ }^{4}$ la elaboración de unas guías para afrontar la incertidumbre y los cambios producidos por la pandemia en el contexto educativo.

Inicialmente, se pretendía preparar dos guías: una guía para ayudar a los maestros a detectar posibles alumnos afectados por el confinamiento (para su derivación al profesional de la psicología de cada centro) y una guía con recomendaciones para las familias sobre cómo ayudar a sus hijos a afrontar la nueva realidad y su reincorporación al colegio en septiembre. La propia dinámica del grupo de trabajo condujo a que la primera de ellas se ampliase al resto de colectivos profesionales que prestan atención educativa (técnicos de rehabilitación, instructores tiflotécnicos...), y con una finalidad no solo de detección, sino ofreciendo en ella algunas herramientas para la intervención. Por otro lado, el grupo de trabajo entendía que el contenido de la guía no podía ser igual si eran padres de niños o si lo eran de adolescentes, por lo que se terminó por elaborar dos guías para padres distintas. Por último, durante la elaboración de la guía para padres de adolescentes, se pensó que había que dirigirse directamente a los propios adolescentes, para lo que se preparó una cuarta guía, orientada hacia la autogestión de sus emociones y la regulación de sus comportamientos ante la nueva realidad, así como para su reincorporación al instituto.

4 Compuesto (por orden alfabético) por Lara Cetina Guede, Félix Dorado Gómez, Jorge González Fernández, Luis González García, Carmen Herrero Menéndez, María José Losada Martínez, Alicia Moreno Jordana, Rafael Pallero González y Eva Vázquez Seoane. 
Así, finalmente, se prepararon cuatro guías, una para profesionales, otra para familias de niños, otra para familias de adolescentes y una cuarta para adolescentes. Todas ellas tenían una estructura similar, y trataban de dar respuesta a las preguntas que a todos nos rondaban por la cabeza: ¿qué había sucedido?, ¿qué habíamos aprendido?, ¿qué nos podía pasar?, ¿qué podíamos hacer?, ¿y si esto no funcionaba?, ¿ya todo seguiría igual? Esta colección de guías, titulada Cómo afrontar la incertidumbre y los cambios debidos a la pandemia, es de difusión interna, entre los profesionales de los equipos de atención educativa de la ONCE, y no está publicada. Las mismas se han trabajado entre los propios profesionales, el alumnado y sus familiares. Además, se les ha ido dando desarrollo desde el seminario sobre ajuste a la discapacidad visual en la infancia y la adolescencia, cuyos grupos de trabajo se coordinan desde los cinco Centros de Recursos Educativos de la ONCE.

Además de estas guías, se subió a ClubONCE el acceso a una colección de diez vídeos (más uno de presentación) titulada Píldoras psicoeducativas para familias durante el confinamiento, albergada en el canal de YouTube del Colegio Oficial de la Psicología de Madrid (s. f.), que contienen consejos prácticos para ayudar a las familias a manejar las dificultades que tienen niños y adolescentes a causa del confinamiento y de la pandemia de la covid-19.

\section{Repercusión de la covid-19 en la salud mental: respuestas desde la psicología}

La llegada de la covid-19 a nuestras vidas ha provocado un notable incremento de la sintomatología de tipo psicológico, principalmente depresión, ansiedad, insomnio, trastorno de estrés postraumático... (American Psychological Association [APA], 2020; Cénat et al., 2021). Sirvan como ejemplo de ese incremento los datos que podemos extraer de varias encuestas recientes: dos de ellas realizadas en España, otra en varios países europeos y la otra en China. Veamos algunos datos que podemos extraer de ellas.

En España, según el Centro de Investigaciones Sociológicas (CIS, 2021), el 79\% de la población manifestaba estar muy o bastante preocupado con la situación de la pandemia de la covid-19. En muchas ocasiones, esa preocupación ha derivado en sintomatología de tipo psicológico. La distribución de dicha sintomatología no es homogénea entre toda la población. Así, si atendemos al sexo de los encuestados, se 
observa que aparece más entre las mujeres que entre los hombres (ver Tabla 1), y, si atendemos a su edad, aparece más cuanto más joven es la población, excepto en el caso de la sintomatología relacionada con la tristeza y la depresión, la cual aumenta a medida que aumenta la edad de los encuestados (ver Tabla 2).

Tabla 1. Porcentaje de personas que presentan muchas o bastantes veces sintomatología psicológica, según su sexo

\begin{tabular}{|l|r|r|}
\hline \multicolumn{1}{|c|}{ Sintomatología psicológica } & Hombres & Mujeres \\
\hline Ansiedad, miedo & $9,5 \%$ & $21,8 \%$ \\
\hline Tristeza, depresión & $11,8 \%$ & $27,7 \%$ \\
\hline Preocupación no controlable & $17,7 \%$ & $31,1 \%$ \\
\hline Soledad, aislamiento & $12,9 \%$ & $21,3 \%$ \\
\hline Desesperanza respecto al futuro & $19,2 \%$ & $30,4 \%$ \\
\hline Irritabilidad, ira, enfado, agresividad & $8,9 \%$ & $18,5 \%$ \\
\hline Nerviosismo & $10,5 \%$ & $24,6 \%$ \\
\hline Agobio, estrés & $14,0 \%$ & $29,3 \%$ \\
\hline Intranquilidad, inquietud & $14,9 \%$ & $29,0 \%$ \\
\hline
\end{tabular}

Fuente: CIS (2021).

Tabla 2. Porcentaje de personas que presentan muchas o bastantes veces sintomatología psicológica, según su edad

\begin{tabular}{|l|r|r|r|}
\hline \multicolumn{1}{|c|}{ Sintomatología psicológica } & $\begin{array}{c}\text { Menores } \\
\text { de 35 años }\end{array}$ & $\begin{array}{c}\text { Entre 35 } \\
\text { y 54 años }\end{array}$ & $\begin{array}{c}\text { Mayores } \\
\text { de 54 años }\end{array}$ \\
\hline Ansiedad, miedo & $36,1 \%$ & $34,8 \%$ & $27,2 \%$ \\
\hline Tristeza, depresión & $34,8 \%$ & $38,3 \%$ & $39,5 \%$ \\
\hline Preocupación no controlable & $65,8 \%$ & $48,6 \%$ & $42,8 \%$ \\
\hline Soledad, aislamiento & $47,3 \%$ & $33,0 \%$ & $29,8 \%$ \\
\hline Desesperanza respecto al futuro & $70,2 \%$ & $51,4 \%$ & $39,3 \%$ \\
\hline Irritabilidad, ira, enfado, agresividad & $37,1 \%$ & $33,1 \%$ & $19,2 \%$ \\
\hline Nerviosismo & $51,0 \%$ & $39,8 \%$ & $25,4 \%$ \\
\hline Agobio, estrés & $66,0 \%$ & $52,4 \%$ & $26,9 \%$ \\
\hline Intranquilidad, inquietud & $63,9 \%$ & $47,2 \%$ & $33,6 \%$ \\
\hline
\end{tabular}

Fuente: cis (2021). 
Resultados similares arroja otra encuesta realizada en España, en este caso en pleno confinamiento domiciliario (Balluerka et al., 2020). Según sus resultados, el 45,7\% de los encuestados habían experimentado un incremento en su malestar psicológico. También es este caso, las mujeres refirieron mayor incremento en el malestar que los hombres (51,8\% frente a 39,4\%). Y, también en este caso, se da una relación inversa entre la edad y el aumento del malestar, de modo que, a menor edad, mayor incremento del malestar (ver Tabla 3).

Tabla 3. Porcentaje de personas que presentan un incremento en su malestar psicológico, según su edad

\begin{tabular}{|l|r|r|r|}
\hline & $\begin{array}{c}\text { Menores } \\
\text { de 35 años }\end{array}$ & $\begin{array}{c}\text { Entre 35 } \\
\text { y 60 años }\end{array}$ & $\begin{array}{c}\text { Mayores } \\
\text { de 60 años }\end{array}$ \\
\hline $\begin{array}{l}\text { Incremento en el malestar } \\
\text { psicológico }\end{array}$ & $53,9 \%$ & $46,3 \%$ & $34,0 \%$ \\
\hline
\end{tabular}

Fuente: Balluerka et al. (2020).

Si ampliamos el foco, una encuesta realizada por la aseguradora AXA (2021) en siete países europeos (Alemania, Bélgica, España, Francia, Italia, Reino Unido y Suiza) señalaba en la misma dirección. Según sus datos, el $23 \%$ de los europeos declaraban que, desde la aparición de la covid-19, su estado mental era malo o muy malo, frente al $8 \%$ que declaraba lo mismo antes de la llegada de la pandemia. Pero la aparición del coronavirus no solo aumentó el porcentaje de los que se sentían mal: también bajó el de los que identificaban su estado mental como bueno o muy bueno (del $60 \%$ al $34 \%$ ).

También en el mismo sentido apuntan los resultados de una encuesta realizada entre la población china, según los cuales más de la mitad de los encuestados refería que la covid-19 les había supuesto un impacto emocional entre moderado y severo, y un tercio informaba de sintomatología ansiosa entre moderada y severa (Wang et al., 2020). Téngase en cuenta que, en este caso, la encuesta fue realizada durante la fase inicial de la pandemia.

En todo el mundo, una parte de las personas que presentaban esa sintomatología generó demandas de apoyo psicológico directo. La respuesta que se dio a esas demandas, tanto desde la ONCE como desde otras instancias, fue inicialmente, durante el confinamiento domiciliario, por medio de la telepsicología (tanto por teléfono como por videoconferencia). Posteriormente, de modo progresivo, se fueron recuperando las atenciones presenciales, aunque sin abandonar la intervención telepsicológica. 
Una parte relevante de las personas, afortunadamente, no llegó a presentar una sintomatología que requiriese de un apoyo psicológico directo, sino que se ha podido beneficiar de las estrategias de tipo psicoeducativo que se desarrollaron en forma de orientaciones y de guías de ayuda emocional.

En lo que a las intervenciones psicológicas por teléfono se refiere, son numerosos los estudios que apoyan la evidencia de su eficacia para abordar la sintomatología ansiosa y depresiva, y ello tanto cuando dichas intervenciones son reiterativas y a medio plazo - una revisión sistemática se puede ver en Leach y Christensen (2006) o, más recientemente, en Vázquez et al. (2015) -, como cuando son una respuesta puntual e inmediata ante crisis agudas (Somer et al., 2005). Además, es de destacar que, como señalan Ribeiro et al. (2020), la intervención psicológica por teléfono «contribuye a una intervención temprana y reactiva, que reduce el aislamiento social y supera diferentes tipos de barreras (por ejemplo, físicas, geográficas, económicas, culturales) [...]. Además, en el escenario pandémico, es conforme con las medidas restrictivas de contacto físico y social, que dificultan los servicios psicológicos tradicionales presenciales» ${ }^{5}$ (p. 4).

En lo que a las intervenciones por videoconferencia se refiere, es importante destacar que las TIC han cambiado nuestra forma de comunicarnos y relacionarnos en sociedad y también están cambiando las formas de provisión de servicios sanitarios y sociales. En este contexto de cambio, la irrupción de la covid-19 en nuestras vidas vino a acelerar este proceso. Siendo grandes los cambios en el cómo se realizan estas intervenciones telepsicológicas, no son menos importantes los cambios relacionados con la proporción que estas intervenciones suponen sobre el total de intervenciones psicológicas. Sirva como referencia una encuesta realizada hace solo cuatro años entre los psicólogos madrileños, la cual indicaba que, en aquel momento, el 26,7\% de ellos utilizaba la terapia por videoconferencia, contemplando su uso en el futuro el 60,5\% (González-Peña et al., 2017). Aún no disponemos de datos sobre el uso que los psicólogos españoles han hecho de la telepsicología para el ejercicio de su profesión durante la crisis de la covid-19, aunque es sobradamente conocido que ha sido muy alto. Sí que disponemos de datos de su uso en Estados Unidos: según una encuesta realizada en septiembre de 2020 por la American Psychological Association (APA), el $96 \%$ de los profesionales estadounidenses de la psicología realizó intervenciones telepsicológicas durante la crisis de la covid-19 (APA, 2020). Nuestros datos son total-

5 Traducción de los autores del original en inglés (N. del E.).

González, L., y Pallero, R. (2021). Intervenciones psicológicas en la ONCE durante la crisis de la covid-19. RED Visual: Revista Especializada en Discapacidad Visual, 77, 68-91. https://doi.org/10.53094/WILG7697. 
mente coincidentes con los de la APA: el 96,7\% de los profesionales de la psicología de la ONCE han hecho intervenciones telepsicológicas durante dicha crisis.

Más allá de esos datos, es interesante realizar un análisis de quiénes son los afiliados a la ONCE a los que se ha prestado apoyo psicológico durante la crisis de la covid-19. Los resultados de la encuesta realizada entre los profesionales de la psicología de la ONCE muestran que dos de cada tres afiliados atendidos por ellos durante la crisis de la covid-19 (en concreto un 62,1\%) tenían entre 30 y 60 años. Sin embargo, el porcentaje de afiliados que se encuentra en ese rango de edad es de menos del $40 \%$. Estos datos indicarían que dicho colectivo estuvo «sobreatendido» en más de 20 puntos porcentuales. De modo complementario, los resultados muestran que una de cada cuatro intervenciones psicológicas (en concreto el 24,1\%) tuvo como destinatarios afiliados de más de 60 años. Ese colectivo supone aproximadamente la mitad del total de los afiliados a la ONCE. Estos datos indicarían que dicho colectivo estuvo «subatendido» en más de 25 puntos. ¿A qué se debe este desfase? ¿Por qué no estamos llegando a los afiliados más mayores, precisamente uno de los colectivos identificados como más vulnerables por parte de la ONCE y, por lo tanto, objeto de especial atención?

Creemos que la respuesta a esas preguntas no es simple, y que ese desfase se debe al efecto combinado de varios factores, entre los que destacaríamos tres:

- Las personas de más edad demandan menos apoyo psicológico. Una encuesta realizada en España (Buela-Casal et al., 2005), informaba que solo el $11 \%$ de las personas mayores de 75 años había acudido alguna vez en su vida al psicólogo, frente al 22,8\% de las personas entre 57-75 años, al 30,2\% de las personas entre 38-56 años, o al 26,8\% de las personas entre 18-37 años. Muchas de ellas optan por una actitud resignada, por pasarlo mal y ocultarlo, antes que reconocer que tienen un problema y necesitan ayuda.

- Las personas de más edad han sido las que menos han visto alterado su bienestar emocional por la covid, como hemos visto en los resultados de las encuestas realizadas en España a las que nos hemos referido anteriormente (CIS, 2021; Balluerka et al., 2020).

- Las personas de más edad son las que menos utilizan Internet y sus servicios asociados. Según la Encuesta sobre equipamiento y uso de tecnologías de in- 
formación y comunicación en los hogares 2020, realizada por el INE (2020), el $83,1 \%$ de la población entre 16 y 74 años utiliza Internet a diario. Pero ese alto porcentaje global esconde una realidad mucho más compleja: los grandes sesgos que se aprecian en función de la edad. Así, ese porcentaje se eleva hasta el $97 \%$ entre la población de 16 a 34 años, pero baja al 50,7\% entre la población de 65 a 74 años. Esas diferencias se acentúan a medida que aumenta la edad de la población: el uso de Internet a diario baja al $21,5 \%$ entre aquellos que tienen de 75 a 84 años, para situarse en un exiguo $7,7 \%$ entre aquellos que tienen 85 y más años. En definitiva: el uso de Internet es inversamente proporcional a la edad de los usuarios.

Este último factor nos señala un elemento que merece una breve reflexión. Según uno de los pocos estudios de los que disponemos (González-Peña et al., 2017), nueve de cada diez profesionales de la psicología (en concreto un $90,8 \%)$ realizaban en 2017 terapia a distancia utilizando la videoconferencia, situándose muy por detrás el uso del teléfono (37,2\%). Sin embargo, la mayoría de las intervenciones realizadas por los profesionales de la psicología de la ONCE durante la crisis de la covid-19 se han realizado combinando la atención por videoconferencia con la atención telefónica (el 58,6\%) o, fundamentalmente, por teléfono (el 27,6\%), mientras que solo el $13,8 \%$ de las intervenciones se realizaron fundamentalmente por videoconferencia. Una de las causas de ese bajo uso de la videoconferencia, frente al mayor uso del teléfono, probablemente la más importante, quizás sea una derivada de uno de los principales problemas que refleja la encuesta realizada entre los profesionales de la psicología de la ONCE: las limitaciones que presentan muchos afiliados en materia de competencia digital. Recordemos que prácticamente cuatro de cada diez profesionales de la psicología de la ONCE perciben la competencia digital de sus usuarios como baja.

En todo caso, siempre debemos tener presente que, como señalan De la Torre y Pardo (2018), el uso de la videoconferencia presenta una serie de ventajas (facilita el acceso a la atención psicológica, es la situación más similar a la intervención presencial, permite distinguir aspectos de la comunicación no verbal, no requiere de una tecnología demasiado específica, permite grabar las sesiones para su análisis...), pero también algunos inconvenientes (solo se ve lo que está en el campo de visión de la cámara, no permite detectar olores u otras señales potencialmente relevantes, requiere una buena cobertura de Internet, a veces se presentan interrupciones en la comunicación, es difícil garantizar la confidencialidad...). 
Lo relacionado con la confidencialidad es especialmente relevante en el caso de las personas afiliadas a la ONCE. Al hecho de que, como acabamos de mencionar, su garantía sea una de las dificultades propias de la videoconferencia (y también de la intervención por teléfono), debemos añadir que, en el caso de las personas con discapacidad visual, la propia discapacidad puede limitar sus posibilidades de verificar la privacidad y confidencialidad de la comunicación. Si a ello añadimos que, como ya se ha mencionado, casi la mitad de los afiliados a la ONCE tienen más de 60 años, y que, como señala el Instituto de Economía Digital, las personas mayores «no tienen interiorizado [sic] los aspectos relacionados con la privacidad y el compartir datos en internet» (Instituto Economía Digital, 2017), convendremos en que estamos ante un punto crítico.

En lo que se refiere a la creación y puesta a disposición de los ciudadanos de materiales para afrontar la crisis de la covid-19, a priori estaríamos ante algo conveniente (Wang et al., 2020), pues este tipo de estrategias, de carácter proactivo, lo que buscan es prevenir el sufrimiento psicológico y promover el bienestar a través del cuidado de los otros y el autocuidado. Así, quizás anticipando el daño emocional que la pandemia iba a suponer (Xiong et al., 2020), quizás por la urgencia que toda pandemia genera (OMS, 2005), probablemente por muchas razones más, profesionales de muchos campos de intervención se lanzaron a preparar materiales para ayudar a la ciudadanía a sobrellevar el confinamiento domiciliario, a mantener el estado físico y emocional, a afrontar el miedo, a vivir el duelo derivado del fallecimiento de sus seres queridos, a volver a una vida cotidiana que tenía poco de cotidiana... Tomemos como referencia a los profesionales españoles de la psicología. Durante la pandemia, el Consejo General de la Psicología de España, y los 23 Colegios Oficiales de la Psicología representados en él, han colgado en sus respectivas páginas web el enlace a casi mil «productos» relacionados con la covid-19 (documentos, vídeos, seminarios web -webinars-, archivos multimedia -podcast-, infografías...). Materiales muy interesantes, creados con muy buena voluntad, elaborados en muy poco tiempo, pero, en algunas ocasiones, repetidos y repetitivos, frecuentemente sin identificar a su autor o autores, y casi nunca sometidos a validación o revisión por pares, y cuyo impacto nunca seremos capaces de conocer. En el caso de los materiales elaborados por los profesionales de la ONCE se habría dado una situación parecida, puesto que, por ejemplo, desconocemos el nivel de uso que se ha hecho de los mismos. Por otro lado, tal vez deberíamos preguntarnos en qué medida esta inflación productiva no ha sido, al menos en parte, uno de los elementos de lo que la socióloga turca Zeynep Tüfekçi (2020) ha denominado «teatro pandémico». 


\section{Reflexiones finales}

En febrero de 2020 la ONCE empezaba a contemplar la posibilidad de realizar intervenciones psicológicas por videoconferencia. La irrupción de la covid-19, en marzo de 2020, supuso que súbitamente, y durante meses, todas las intervenciones psicológicas se tuvieran que realizar mediante telepsicología (videoconferencia y teléfono). Con el paso de los meses, la telepsicología se ha convertido en una vía más de intervención, experimentando un crecimiento exponencial a lo largo de 2020.

Debemos estar atentos a que este incremento en el uso de la telepsicología, especialmente de la realizada por videoconferencia, no deje al margen a las personas con menos destrezas en el manejo de las TIC. En este sentido, entendemos que es esencial atender las necesidades de formación que puedan presentar algunos usuarios, especialmente aquellos más mayores y menos habituados a su uso. Esta tarea debería ser desarrollada de modo colaborativo, con una especial participación de los instructores tiflotécnicos.

En cuanto a las intervenciones psicológicas realizadas directamente con los afiliados, creemos que su contribución a la mejora de su calidad de vida, tanto durante las semanas de confinamiento domiciliario como después, ha sido muy positiva, aunque no disponemos de datos cuantitativos que avalen esta estimación.

Es de destacar el importante y productivo trabajo en red realizado por los profesionales de la psicología de la ONCE, quienes han revisado y valorado decenas de documentos y otros materiales producidos por diversas entidades, al tiempo que ellos mismos han elaborado diversos materiales para uso de los afiliados y sus familiares. Consideramos que este trabajo también ha tenido un impacto favorable en la mejora de la calidad de vida de los afiliados, aunque tampoco disponemos de datos cuantitativos que avalen esta estimación. En todo caso, esta capacidad para trabajar en red entendemos que es la base sobre la que se pueden asentar futuros trabajos colaborativos, aplicables a todo tipo de situaciones.

Tanto en el caso de las intervenciones psicológicas (presenciales, por teléfono o por videoconferencia) como en las intervenciones psicoeducativas, sería importante poder conocer la relación entre el esfuerzo de los profesionales y su incidencia en los usuarios, así como el valor que estos atribuyen a dichas intervenciones. 


\section{Referencias bibliográficas}

American Psychological Association (2020). Patients with depression and anxiety surge as psychologists respond to the coronavirus pandemic. American Psychological Association. https://www.apa.org/news/press/releases/2020/11/telehealth-survey-summary.pdf.

Anderson, C. M., Hogarty, G. E., y Reiss, D. J. (1980). Family treatment of adult schizophrenic patients: a psycho-educational approach [PDF]. Schizophrenia Bulletin, 6(3), 490-505. https://doi.org/10.1093/schbul/6.3.490.

Authier, J. (1977). The psychoeducation model: definition, contemporary roots and content [PDF]. Canadian Journal of Counselling and Psychotherapy, 12(1), 15-22.

AXA (2020). A report on mental health \& wellbeing in Europe [PDF]. AXA. https://www. axa.com/en/press/publications/A-Report-on-Mental-Health-and-Wellbeing-in-Europe.

Balluerka, N., Gómez-Benito, J., Hidalgo, M.D., Gorostiaga, A., Espada, J.P., Padilla, J.L., y Santed, M.A. (2020). Las consecuencias psicológicas de la COVID-19 y el confinamiento: informe de investigación [PDF]. Servicio de Publicaciones de la Universidad del País Vasco.

Buela-Casal, G., Teva, I., Sierra, J.C., Bretón-López, J., Agudelo, D., Bermúdez, M.P., y Gil Roales-Nieto, J. (2005). Imagen de la psicología como profesión sanitaria entre la población general [PDF]. Papeles del Psicólogo, 26(91), 30-38.

Cénat, J.M., Blais-Rochette, C., Kokou-Kpolou, C.K., Noorishad, P.-G., Mukunzi, J.N., McIntee, S.-E., Dalexis, R.D., Goulet, M.-A., Labelle, P.R. (2021). Prevalence of symptoms of depression, anxiety, insomnia, posttraumatic stress disorder, and psychological distress among populations affected by the COVID-19 pandemic: a systematic review and metaanalysis. Psychiatry Research, 295, 113599. https://doi.org/10.1016/j.psychres.2020.113599.

Centro de Investigaciones Sociológicas (2021). Encuesta sobre la salud mental de los españoles durante la pandemia de la COVID-19: avance de resultados. http://www.cis. es/cis/opencms/ES/NoticiasNovedades/InfoCIS/2021/Documentacion_3312.html.

Colegio Oficial de la Psicología de Madrid (s. f.). Píldoras psicoeducativas para familias durante el confinamiento [vídeos en YouTube]. https://www.youtube.com/playlist?list= PLIJsbJFGKZG1ZpVqaXK-ECqTHp3G5XGsv. 
González-Peña, P., Torres, R., Barrio, V. del, y Olmedo, M. (2017). Uso de las nuevas tecnologías por parte de los psicólogos españoles y sus necesidades [PDF]. Clínica y Salud, 28(2), 81-91. https://doi.org/10.1016/j.clysa.2017.01.001.

Instituto Economía Digital (2017). Las 6 generaciones de la era digital. https://cdn5.icemd. com/app/uploads/2018/12/Estudio_6-generaciones-de-la-era-digital-.pdf.

Instituto Nacional de Estadística (2020). Encuesta sobre equipamiento y uso de tecnologías de información y comunicación en los hogares. https://www.ine.es/dynt3/inebase/es/ index.htm?padre=6899\&capsel=6900.

Leach, L. S., y Christensen, H. (2006). A systematic review of telephone-based interventions for mental disorders. Journal of Telemedicine and Telecare, 12(3), 122-129. https://doi. org/10.1258/135763306776738558.

Lemes, C.B., y Ondere, J. (2017). Aplicações da psicoeducação no contexto da saúde [PDF]. Temas em Psicologia, 25(1), 17-28. https://doi.org/10.9788/TP2017.1-02.

Organización Mundial de la Salud (s. f.). Naming the coronavirus disease (COVID-19) and the virus that causes it. https://www.who. int/emergencies/diseases/novel-coronavirus-2019/ technical-guidance/naming-the-coronavirus-disease-(covid-2019)-and-the-virus-thatcauses-it.

Organización Mundial de la Salud (2005). WHO outbreak communication guidelines [PDF]. Organización Mundial de la Salud.

Organización Mundial de la Salud (5 de febrero de 2020). Novel coronavirus (2019-nCoV). Situation report [PDF]. Organización Mundial de la Salud.

Organización Mundial de la Salud (11 de marzo de 2020). WHO Director-General's opening remarks at the media briefing on COVID-19 - 11 March 2020. https://www.who.int/directorgeneral/speeches/detail/who-director-general-s-opening-remarks-at-the-media-briefingon-covid-19---11-march-2020.

Real Decreto 463/2020, de 14 de marzo, por el que se declara el estado de alarma para la gestión de la situación de crisis sanitaria ocasionada por el CoVID-19 [PDF]. Boletín Oficial del Estado, 67, 14 de marzo de 2020, 25390-25400. 
Ribeiro, E., Sampaio, A., Gonçalves, M.M., Taveira, M.C., Cunha, J., Maia, Â., Matos, M., Gonçalves, S., Figueiredo, B., Freire, T., y Soares, T. (2020) Telephone-based psychological crisis intervention: the Portuguese experience with COVID-19 [PDF]. Counselling Psychology Quarterly, 7 de junio de 2020. https://doi.org/10.1080/09515070.2020.1772200.

Somer, E., Tamir, E., Maguen, S., y Litz, B.T. (2005). Brief cognitive-behavioral phonebased intervention targeting anxiety about the threat of attack: a pilot study. Behaviour Research and Therapy, 43(5), 669-679. https://doi.org/10.1016/j.brat.2004.05.006.

Torre, M. de la, y Pardo, R. (2018), Guía para la intervención telepsicológica [PDF]. Colegio Oficial de Psicólogos de Madrid.

Tüfekçi, Z. (7 de abril de 2020). Keep the parks open. The Atlantic. https://www.theatlantic. com/health/archive/2020/04/closing-parks-ineffective-pandemic-theater/609580.

Vázquez, F. L., Torres, Á., Blanco, V., Otero, P., y Hermida, E. (2015). Intervenciones psicológicas administradas por teléfono para la depresión: una revisión sistemática y meta-análisis [PDF]. Revista Iberoamericana de Psicología y Salud, 6(1), 39-52. https:// doi.org/10.1016/S2171-2069(15)70005-0.

Wang, C., Pan, R., Wan, X., Tan, Y., Xu, L., Ho, C.S., y Ho, R.C. (2020). Immediate psychological responses and associated factors during the initial stage of the 2019 coronavirus disease (COVID-19) epidemic among the general population in China [PDF]. International Journal of Environmental Research and Public Health 17(5), 1729. https://doi.org/10.3390/ijerph17051729.

Xiong, J., Lipsitz, O., Nasri, F., Lui, L. M.W., Gill, H., Phan, L., Chen-Li, D., Iacobucci, M., Ho, R., Majeed, A., y McIntyre, R.S. (2020). Impact of COVID-19 pandemic on mental health in the general population: a systematic review. Journal of Affective Disorders, 277, 55-64. https://doi.org/10.1016/j.jad.2020.08.001.

Luis González García. Psicólogo. Coordinador del grupo de trabajo sobre telepsicología en la ONCE. Dirección de la ONCE en Vigo. Avenida da Gran Vía, 16; 36203 Vigo, Pontevedra (España). Correo electrónico: lugg@once.es.

Rafael Pallero González. Psicólogo. Asesor de la Dirección de Autonomía Personal. Dirección de la ONCE en Tarragona. Rambla Vella, 10; 43003 Tarragona (España). Correo electrónico: rpgo@once.es. 